\title{
Is CRP-guided antibiotic treatment a safe way to reduce antibiotic use in severe hospitalised patients with exacerbations of COPD?
}

To the Editor:

We read with great interest the well-conducted clinical trial on C-reactive protein (CRP)-guided antibiotic treatment in admitted patients with acute exacerbations of COPD by PRINs et al. [1]. This is a study showing that the use of CRP can potentially assist clinicians in making more prudent use of antibiotics in patients with severe exacerbations, as has also been found in the primary care setting [2]. One of the striking results is, however, the disturbingly high rate of treatment failure observed of approximately $24 \%$ at 10 days and $45 \%$ at 30 days, as pointed out in the accompanying editorial [3], and consistent with this high rate of treatment failure is a remarkably short time to next exacerbation of less than 1 month [1]. Since the percentage of patients treated with antibiotics in both study groups was very low ( $31 \%$ and $46 \%$ ), it would be necessary to rule out that the reduction in the use of antibiotics is not responsible for an increase in clinical failures in this population with severe exacerbations of COPD. For comparison, a recent study using CRP to guide antibiotic use in much milder COPD patients in an ambulatory setting resulted in $57 \%$ of patients treated with antibiotics [4], in contrast to the $31 \%$ antibiotic prescription in CRP guided therapy in severe hospitalised patients in the study by Prins et al. [1].

Interestingly, the main outcome in the study by PRINS et al. [1] is not a clinical outcome, but the reduction in the use of antibiotics with the CRP strategy compared to the Global Initiative for Chronic Obstructive Lung Disease (GOLD) strategy. This would be appropriate if both strategies for reduction of antibiotic use in admitted COPD patients would have been fully validated for efficacy and safety. However, there is no information in the article about the comparison of failure rates of patients taking or not taking antibiotics as a result of the application of either strategy. This information is crucial for the interpretation of the results, because it is very easy to restrict the use of antibiotics to patients with a CRP $>50 \mathrm{mg} \cdot \mathrm{L}^{-1}$, but it must be demonstrated that hospitalised patients not taking antibiotics due to a CRP $<50 \mathrm{mg} \cdot \mathrm{L}^{-1}$ are not at increased risk of failure due to this decision.

In order to have a complete understanding of the results and the clinical implications of their interesting study it would be necessary to have information about the clinical outcomes (30-day treatment failure, time to the next exacerbation and length of stay) in patients in whom antibiotics were prescribed compared to those not taking antibiotics in both study groups. This information would better clarify whether the desirable reduction of antibiotic use observed in the CRP-guided group was associated or not with an increased risk of clinical failure.

Antibiotics have differential effects on clinical outcomes based on their pharmacodynamic/pharmacokinetic properties. In severe exacerbations, antibiotic resistant pathogens such as Pseudomonas aeruginosa are common. There is no information provided whether amoxicillin/clavulanate, the primary antibiotic of choice in this study provided adequate coverage of the pathogens isolated from sputum in the participants. Bacteriological eradication of pathogens at exacerbation has been associated with clinical outcomes [5]. It is possible that inadequate antibiotic therapy was responsible for poor clinical outcomes in the antibiotic arms in this study.

It has been demonstrated that nearly $60 \%$ of patients with ambulatory exacerbations of mild-to-moderate COPD can be cured without antibiotics $[6,7]$, which means that we have substantial room for improvement in

@ERSpublications

A reduction in antibiotic use to treat COPD must be safe for the patient http://bit.ly/2GxGpTO

Cite this article as: Miravitlles M, Llor C, Bjerrum L, et al. Is CRP-guided antibiotic treatment a safe way to reduce antibiotic use in severe hospitalised patients with exacerbations of COPD? Eur Respir J 2019; 54: 1901405 [https://doi.org/10.1183/13993003.01405-2019]. 
antibiotic prescription in this population. Furthermore, a series of variables, including CRP, may be useful to identify patients with exacerbations who can be safely treated without antibiotics in an ambulatory setting $[4,8]$. These data indicate that a drastic reduction of antibiotic use is possible and needed in the outpatient setting, in which exacerbations are usually milder and self-limiting, and a significant number can be non-infective. However, we must be much more careful in reducing the use of antibiotics in severe exacerbations, which are associated with a poor short- and long-term prognosis if not adequately treated $[9,10]$.

PRINS et al. [1] suggest that CRP-guided antibiotic therapy is able to reduce antimicrobial pressure while maintaining patient safety. This is true for the comparison between this strategy and the GOLD strategy. However, patient safety should be demonstrated by comparing the outcomes of patients with and without antibiotics as a result of the application of the CRP strategy. Having this additional information would better help clinicians to understand if the CRP-guided strategy may be a good instrument to safely reduce antimicrobial use in admitted patients with exacerbations of COPD.

Marc Miravitlles ${ }^{1}{ }^{1}$, Carl Llor $^{2}$, Lars Bjerrum ${ }^{2}$, Sanjay Sethi ${ }^{3}$ and Antonio Anzueto ${ }^{4,5}$

${ }^{1}$ Pneumology Dept, Hospital Universitari Vall d'Hebron/Vall d'Hebron Research Institute (VHIR), CIBER de Enfermedades Respiratorias (CIBERES), Barcelona, Spain. ${ }^{2}$ Research Unit for General Practice and Section of General Practice, Dept of Public Health, University of Copenhagen, Copenhagen, Denmark. ${ }^{3}$ Division of Pulmonary, Critical Care and Sleep Medicine, Dept of Medicine, University at Buffalo, SUNY, Buffalo, NY, USA. ${ }^{4}$ Division of Pulmonary Diseases and Critical Care Medicine, School of Medicine, The University of Texas Health Science Center, San Antonio, TX, USA. ${ }^{5}$ Audie L. Murphy Memorial VA Hospital, South Texas Veterans Health Care System, San Antonio, TX, USA.

Correspondence: Marc Miravitlles, Pneumology Dept, Hospital Universitari Vall d'Hebron, P. Vall d'Hebron 119-129, 08035 Barcelona, Spain. E-mail: marcm@separ.es

Received: 15 July 2019 | Accepted: 20 July 2019

Conflict of interest: M. Miravitlles reports personal fees for lectures from AstraZeneca, Boehringer Ingelheim, Chiesi, Cipla, Menarini, Rovi, Bial, Zambon, CSL Behring, Grifols and Novartis, personal fees for consultancy from AstraZeneca, Boehringer Ingelheim, Chiesi, GlaxoSmithKline, Bial, Gebro Pharma, CSL Behring, Laboratorios Esteve, Ferrer, Mereo Biopharma, Verona Pharma, TEVA, pH Pharma, Novartis and Grifols, grants from GlaxoSmithKline and Grifols, outside the submitted work. C. Llor reports grants from Abbott Diagnostics, outside the submitted work. L. Bjerrum has nothing to disclose. S. Sethi reports grants and personal fees from GlaxoSmithKline and AstraZeneca, personal fees from Boehringer Ingelheim, Bayer, Aradigm, Sunovion and Theravance, outside the submitted work. A. Anzueto reports personal fees for consultancy from GlaxoSmithKline, AstraZeneca and Boehringer Ingelheim, outside the submitted work.

\section{References}

1 Prins HJ, Duijkers R, van der Valk P, et al. CRP-guided antibiotic treatment in acute exacerbations of COPD in hospital admissions. Eur Respir J 2019; 53: 1802014.

2 Aabenhus R, Jensen JUS, Jørgensen KJ, et al. Biomarkers as point-of-care tests to guide prescription of antibiotics in patients with acute respiratory infections in primary care. Cochrane Database Syst Rev 2014; 11: CD010130.

3 Schuetz P, Stolz D. Guiding antibiotic treatment with inflammatory biomarkers in COPD? Another brick in the wall. Eur Respir J 2019; 53: 1900562.

4 Butler CC, Gillespie D, White P, et al. C-Reactive protein testing to guide antibiotic prescribing for COPD exacerbations. N Engl J Med 2019; 381: 111-120.

5 Wilson R, Anzueto A, Miravitlles M, et al. Moxifloxacin vs amoxicillin/clavulanic acid in acute exacerbations of COPD: MAESTRAL study results. Eur Respir J 2012; 40: 17-27.

6 Llor C, Moragas A, Hernández S, et al. Efficacy of antibiotic therapy for acute exacerbations of mild to moderate chronic obstructive pulmonary disease. Am J Respir Crit Care Med 2012; 186: 716-723.

7 Wedzicha JA, Miravitlles M, Hurst JR, et al. Management of COPD exacerbations: a European Respiratory Society/American Thoracic Society guideline. Eur Respir J 2017; 49: 1600791.

8 Miravitlles M, Moragas A, Hernández S, et al. Is it possible to identify exacerbations of mild to moderate COPD that do not require antibiotic treatment? Chest 2013; 144: 1571-1577.

9 Alcázar Navarrete B, Ancochea Bermúdez J, García-Río F, et al. Patients with chronic obstructive pulmonary disease exacerbations: recommendations for diagnosis, treatment and care. Arch Bronconeumol 2019; 55: 478-487.

10 Wageck B, Cox NS, Holland AE. Recovery following acute exacerbations of chronic obstructive pulmonary disease a review. COPD 2019; 16: 93-103.

Copyright CERS 2019

From the authors:

We thank M. Miravitlles and colleagues for their interest in our work [1]. They express concern about our failure rate: $24 \%$ at 10 days, and $45 \%$ at day 30 ; they feel that $31 \%$ of patients of the C-reactive protein (CRP) group and $46 \%$ of patients of the Global Initiative for Chronic Obstructive Lung Disease (GOLD) strategy group treated with antibiotics for acute COPD exacerbation is low in this high-risk population of 
TABLE 1 Antimicrobial prescription and outcome stratified according to Global Initiative for Chronic Obstructive Lung Disease (GOLD) and C-reactive protein (CRP) guidance

\begin{tabular}{|c|c|c|c|c|c|c|}
\hline & \multicolumn{3}{|c|}{ Antimicrobial treatment $(n=87)$} & \multicolumn{3}{|c|}{ No antimicrobials ( $n=133)$} \\
\hline & $\begin{array}{c}\text { GOLD group } \\
\text { (n=55) }\end{array}$ & $\begin{array}{c}\text { CRP group } \\
(n=32)\end{array}$ & p-value & $\begin{array}{c}\text { GOLD group } \\
(n=64)\end{array}$ & $\begin{array}{l}\text { CRP group } \\
{[n=69)}\end{array}$ & p-value \\
\hline 30-day treatment failure rate & 10 (31.3) & 17 (30.9) & 0.974 & $36(52.2)$ & $36(56.3)$ & 0.637 \\
\hline Time to next exacerbation days & $34(22-72)$ & $55(15-121)$ & 0.761 & $19(7-68)$ & $17(6-53)$ & 0.792 \\
\hline Length of stay days & $6(5-8)$ & $6(5-9)$ & 0.933 & $7(4-11)$ & $6(4-9)$ & 0.077 \\
\hline
\end{tabular}

Data are presented as $\mathrm{n}(\%)$ or median (interquartile range), unless otherwise stated.

hospitalised patients. Although treatment failure is high in our study, it reflects the severity of our population. Indeed, the proportion of patients on antimicrobials is lower than the outpatient study population in a recently published trial from the UK [2]; however, our COPD population is more severe and consists of hospitalised patients. Their concern is safety: have we caused harm in our patients by withholding antimicrobial treatment? First, in our study population, there was no significant difference in failure rates at days 10 and 30 between the CRP and GOLD group, which strongly argues against their point that antimicrobial treatment might have prevented harmful events (table 1). Neither failure during admission, nor relapse was significantly different between both study arms. Indeed, relapses among patients with acute exacerbation of COPD admitted to hospital are common [3], especially among individuals with a low forced expiratory volume in $1 \mathrm{~s}$, but antimicrobial treatment may not necessarily prevent this, in particular among those that had low inflammatory markers. Slow recovery and early relapse have also been associated with increased inflammation, e.g. reflected by persistently increased CRP, and in patients characterised by chronic bronchitis [4], but whether these individuals might benefit from antimicrobial treatment if their CRP is below a given threshold has not been addressed in clinical studies [5]. An earlier study suggested that patients with CRP $>50 \mathrm{mg} \cdot \mathrm{L}^{-1}$ benefit more from antibiotic treatment compared to patients with CRP below this threshold [6]. Second, they argue that perhaps the active study arm treated with co-amoxiclav as the primary antimicrobial agent might have been inadequate. Although antimicrobial susceptibility data have not been listed in our paper, Pseudomonas spp. or other high-risk pathogens were covered if retrieved from sputum, and patients known to have colonisation with high-risk pathogens were provided with tailored antimicrobial regimens.

Third, we agree with M. Miravitlles and colleagues that our study was not powered to demonstrate safety beyond all reasonable doubt for patients in whom antimicrobial treatment was witheld based on the CRP decision rule alone [7]. Besides the initial reduction of antibiotics of more than $30 \%$ (from $46.2 \%$ to $31.7 \%$ ) associated with the CRP algorithm, around $30 \%$ of the patients were additionally treated with antibiotics due to treatment failure (equally distributed between the two groups). Importantly, this did not result in an increase of adverse events or length of hospital stay.

Our study provides preliminary data suggesting safety, and therefore argues in favour of a larger international multicentre trial to address this question more definitively for patients with COPD exacerbation that are hospitalised.

Antimicrobial treatment may cause serious harm, first of all, for individuals themselves [8]. Differences across geographic regions suggest that outpatient antimicrobial prescription is at least in part culturally, not scientifically triggered [9]. Indeed Spain, Cyprus and Mongolia do worse than some other locales, e.g. the Netherlands. If we fail to reduce our antimicrobial footprint, sooner or later we will lose the war on antimicrobial resistance [10].

@ERSpublications

CRP-guided antibiotic treatment reduces antibiotic prescription in acute exacerbation without change in treatment failure http://bit.ly/33IweWG

Cite this article as: Prins H-J, van der Werf TS, Boersma WG. Is CRP-guided antibiotic treatment a safe way to reduce antibiotic use in severe hospitalised patients with exacerbations of COPD? Eur Respir J 2019; 54: 1901597 [https://doi.org/10.1183/13993003.01597-2019]. 
Henk-Jan Prins ${ }^{1}$, Tjip S. van der Werf ${ }^{2}$ and Wim G. Boersma ${ }^{1}$

${ }^{1}$ Dept Pulmonary Diseases, Northwest Hospital, Alkmaar, The Netherlands. ${ }^{2}$ Dept of Pulmonary Diseases and Tuberculosis, University of Groningen, University Medical Center, Groningen, The Netherlands.

Correspondence: H-J. Prins, Northwest Hospital, Pulmonary Diseases, Wilhelminalaan 12, Alkmaar, 1815JD, The Netherlands. E-mail: hjprins1982@hotmail.com

Received: 10 Aug 2019 | Accepted: 13 Aug 2019

Conflict of interest: H-J. Prins has nothing to disclose. T.S. van der Werf has nothing to disclose. W.G. Boersma reports grants from GSK and Foreest Medical School, during the conduct of the study.

\section{References}

1 Prins HJ, Duijkers R, van der Valk P, et al. CRP-guided antibiotic treatment in acute exacerbations of COPD in hospital admissions. Eur Respir J 2019; 53: 1802014.

2 Butler CC, Gillespie D, White P, et al. C-Reactive protein testing to guide antibiotic prescribing for COPD exacerbations. N Engl J Med 2019; 381: 111-120.

3 Cao Z, Ong KC, Eng P, et al. Frequent hospital readmissions for acute exacerbation of COPD and their associated factors. Respirology 2006; 11: 188-195.

4 Wageck B, Cox NS, Holland AE. Recovery following acute exacerbations of chronic obstructive pulmonary disease a review. COPD 2019; 16: 93-103.

5 Ko FW, Chan KP, Hui DS, et al. Acute exacerbation of COPD. Respirology 2016; 21: 1152-1165.

6 Daniels JM, Schoorl M, Snijders D, et al. Procalcitonin vs C-reactive protein as predictive markers of response to antibiotic therapy in acute exacerbations of COPD. Chest 2010; 138: 1108-1115.

7 Schuetz P, Stolz D. Guiding antibiotic treatment with inflammatory biomarkers in COPD? Another brick in the wall. Eur Respir J 2019; 53: 1900562.

8 Shehab N, Patel PR, Srinivasan A, et al. Emergency department visits for antibiotic-associated adverse events. Clin Infect Dis 2008; 47: 735-743.

9 Goossens H, Ferech M, Vander Stichele R, et al. Outpatient antibiotic use in Europe and association with resistance: a cross-national database study. Lancet 2005; 365: 579-587.

10 Limmathurotsakul D, Sandoe JAT, Barrett DC, et al. 'Antibiotic footprint' as a communication tool to aid reduction of antibiotic consumption. J Antimicrob Chemother 2019; 74: 2122-2127. 